\title{
Teaching first order phase transition using a zipper model
}

\author{
Reshma Perayil ${ }^{1 \text { a. * }}$, Kandoth Murkoth Udayanandan ${ }^{2 b}$, Prasanth Pulinchery ${ }^{3 \mathrm{c}}$ \\ ${ }^{1}$ S N Polytechnic College, Kanhangad, 671315, India \\ 2 Former HOD, NAS College, Kanhangad, 671315, India \\ ${ }^{3}$ Govt. Engineering College, Thrissur, 680009, India \\ a reshmaperayil@gmail.com; b udayanandan@gmail.com; c prasanthpnairg@gmail.com \\ * Corresponding Author.
}

Received: 10 August 2021; Revised: 8 October 2021; Accepted: 6 November 2021

\begin{abstract}
In 1968, Kittel published the zipper model for demonstrating the first order phase transition which is applicable to DNA like structures. This paper utilize this model to explain the behavior of many thermodynamic properties before, at and after phase transition to get a clear picture about what the changes are happening to the system during phase transition. Many textbooks say that entropy and volume show discontinuity at phase transition whereas Gibbs free energy is continuous. The zipper model shows that energy is also discontinuous as shown in the paper. Understanding the phase transition is still a difficult topic for the undergraduate students and hopefully this paper can help them to overcome this difficulty.

Keywords: Zipper model; Statistical Mechanics; First order phase transition
\end{abstract}

How to Cite: Perayil, R., Murkoth, U. K., \& Pulinchery, P. (2022). Teaching first order phase transition using a zipper model. Momentum: Physics Education Journal, 6(1), 1-9. https://doi.org/10.21067/mpej.v6i1.5850

\section{Introduction}

Materials around us exist in various forms and the changes in their environment lead to the changes in their properties, consider the case of water which is heated up to a temperature of $100^{\circ} \mathrm{C}$ from room temperature at atmospheric pressure. A small change from below $100^{\circ} \mathrm{C}$ above make the volume of water changes dramatically and it transforms to vapor, which is the most common example of phase transition. As we look around, we see that not only there are different phases of matter but they undergo transformation from one phase to another under different conditions.

The history traces back to 1822 when Charles Cagniard de la Tour studied the transformation in alcohol and characterized the transition between liquid and gas phase at different temperature, pressure and volume (Ali, 2021), Subsequently, in 1863, Thomas Andrews carried out his experimental work with a large number of liquids and coined the term "critical point" referring to the point at which transformation takes place. In 1873, Josiah Willard Gibbs came out with a phase diagram, a graph showing different regions of phase characterized by distinct temperature and pressure (Xiong et al., 2011). Weiss in 1907 derived the location of critical point where the ferromagnetism disappears in the case of magnetic material (Koksharov, 2020). Weiss and Kamerlingh Onnes named the critical point as "Curie point" in 1910 (Gridnev \& Popov, 2020; Shamis \& Zeitouni, 2018). By 1922, W.H. Keesom and his coworkers in the low temperature laboratory at University of Leiden, conducted a series of measurement on the dielectric constant of the liquid helium suggesting the occurrence of a phase transition (Banuti et al., 2017). Keesom and Clausius observed that the heat capacity increases to an infinite value and then jump to much lower value about one third of the maximum. They constructed a discontinuous curve to the data and named it "lambda transition". Ehrenfest, in his mathematical paper, explained the difference between the observed helium transition and the transitions observed earlier (Sauer, 2017). As the history predict 
the occurrence of phase transition, physicist started thinking in a broader sense and started applying his understanding to a wide range of systems. The ideas of statistical mechanics have been incorporated to classify them as first or second order and to analyze the thermodynamic properties.

Statistical mechanics is a branch of physics which use the principles of probability for finding the thermodynamics of systems (Oono, 2017). Three important thermodynamic ensembles(Inaba, 2015) are; Micro canonical ensemble (MCE): A statistical ensemble where the energy, volume and the number of particles in the system are fixed, and hence can be a completely isolated system, Canonical ensemble (CE): An ensemble where temperature, volume and the number of particles is fixed, a system which is in contact with a heat bath so that energy is exchanged, and Grand canonical ensemble (GCE): An ensemble where the temperature, volume and chemical potential are fixed, a system that is in contact with both a heat bath and a particle bath. All ensembles give same result but we consider canonical ensemble for further discussion because of its simplicity. The recipe of thermodynamics in canonical ensemble (Bagchi, 2018) is:

$$
\begin{aligned}
& \mathrm{U}=-\left[\frac{\partial}{\partial \beta} \ln \mathrm{Q}_{\mathrm{N}}\right]_{\mathrm{V}, \mathrm{N}} \\
& <\mathrm{s}>=-\frac{1}{\epsilon}\left[\frac{\partial}{\partial \beta} \ln \mathrm{Q}_{\mathrm{N}}\right]_{\mathrm{V}, \mathrm{N}}
\end{aligned}
$$

$\mathrm{F}=-\mathrm{k} \mathrm{T} \ln \mathrm{Q}_{\mathrm{N}}$

$S=-\left[\frac{\partial F}{\partial T}\right]_{V, N}$

$\mathrm{G}=\mathrm{F}-\mathrm{TS}$

where $U$ is the average energy, $\beta=\frac{1}{k T}, \mathrm{k}$ is the Boltzmann constant, $\mathrm{T}$ is the absolute temperature, $\langle s\rangle$ is the average number of links in the zipper which we will be discussing later, $F$ is the Helmholtz free energy and $G$ is the Gibbs free energy.

Our goal in this paper is to study a model called zipper as a means of identifying the key features of a first order phase transition. Zipper, a simple statistical physics model of the long chain of links formation is an interesting member of the class of one-dimensional systems, which exhibits a first order phase transition (Bellantuono et al., 2019; Finkelstein \& Ptitsyn, 2016). Gibbs and DiMarzio were the first to discuss about double ended zipper in which unwinding is permitted from both the ends (Kojima et al., 2015; Park, 2020). The uncoiling of the helical conformation of polypeptide chain and the separation of the two strands of the double stranded helix of deoxyribonucleic acid (DNA) were discussed as two problems in statistical mechanics (Brahmachari \& Marko, 2018). In long lengths of DNA and in related synthetic polymers, the two strands may separate in region as well at their ends, so that zipper model is over simplified. The single ended zipper can be treated as simpler than any related problem, and it offers a good way to introduce biophysics and chemistry into a course on statistical physics (Naiser et al., 2008; Zeng et al., 2021; Zhu et al., 2019).

\section{Methods}

Here we consider a zipper of $\mathrm{N}$ links that can be opened only from one end as in Figure 1 . The last link $\mathrm{N}$ cannot be opened and hence zipper is completely open only when ( $\mathrm{N}-1$ ) links are open (Deger et al., 2018). Each open state of the link is $\Omega$-fold generate, corresponding to the rotational freedom of a link. If $\mathrm{N}$ links are open the total degeneracy is $\Omega^{N}$.

\section{Partition function}

Zipper has two phases. First one is the completely closed state, which represents the phase with temperature $T<T_{c}$, where $T_{c}$ is the critical temperature at which phase transition starts (Deger \& Flindt, 2019; Mogliacci et al., 2019). Second phase of the zipper begins when one link is in 
open state, and this can be considered as $T=T_{c}$ state. If more links are open, we can say $T>T_{c}$ and if all links are open, we can say $T \gg T_{c}$. This is the second phase of zipper. Hence for a zipper there are 3 situations, $T<T_{c}, T=T_{c}$ and $T>T_{c}$, for which the partition functions will be different since the energies available will be different (Badasyan, 2021; Janke et al., 2017).

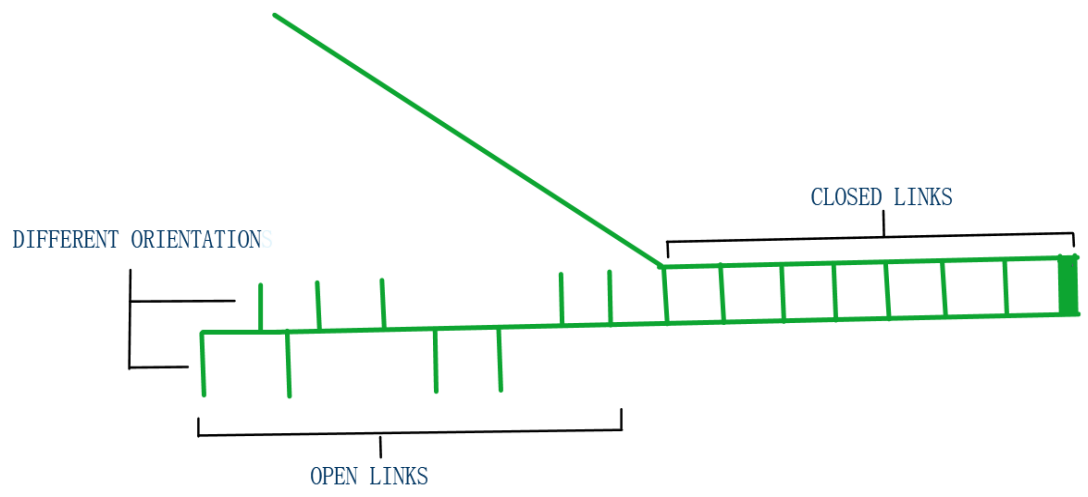

Figure 1. Molecular zipper

Let $\epsilon$ be the energy required to open a link. In the first case when all the links are closed $\epsilon=0$ and this is the case for temperature $T \ll T_{c}$. Since only the open link has a degeneracy, here $\Omega=1$. Hence the partition function is

$Q_{N}=\Sigma \Omega^{N} e^{-\beta N \epsilon}=1$

which gives

$\ln Q_{N}=0$

And hence all the thermodynamics will be zero. In the second situation for $T=T_{c}$ one closed state changes to an open state with energy equal to $\epsilon$. Then the partition function becomes

$Q_{N}=\Omega^{N}\left[1+e^{-\beta \epsilon}\right]^{N}$

and hence

$\ln Q_{N}=N \ln \left[\Omega\left(1+e^{-\beta \epsilon}\right)\right]$

For $T>T_{c}$ more than one link is open with each of energy $\epsilon$. Hence to open $p$ links energy is $p \in$ and the total degeneracy is $\Omega^{p}$. We assume that the last link of the zipper will never be open and therefore the summation is considered only up to $\mathrm{N}-1$, not $\mathrm{N}$. The partition function is,

$Q=\sum_{p=0}^{N-1} \Omega^{P} e^{-\beta p \epsilon}=\sum_{p=0}^{N-1}\left[\Omega e^{-\beta \epsilon}\right]^{p}$

Since $\epsilon, \beta$ are all positive, the exponent will always be less than or equal to one. We can turn to the geometric series

$$
\sum_{a=0}^{N-1} r^{a}=\frac{1-r^{N}}{1-r}
$$

To simplify the equation. Thus, our partition function can be rewritten as

$$
Q_{N}=\frac{1-\Omega^{N} e^{-\beta N \epsilon}}{1-\Omega e^{-\beta \epsilon}}
$$


Momentum: Physics Education Journal, 6 (1), 2022, 4

Reshma Perayil, Kandoth Murkoth Udayanandan, Prasanth Pulinchery

Let $x=\Omega e^{-\beta \epsilon}$. Then the partition function becomes,

$Q_{N}=\frac{1-x^{N}}{1-x}$

Now we will find the thermodynamic quantities for finite $\mathrm{N}$ and then examine them in the large N limit (Fultz, 2020; Thiele et al., 2019).

\section{Results and Discussions}

The Phase Transition

Using the formulas 1 to 5 we get the energy(U), average number of links ( $<\mathrm{s}>$ ), entropy(S) and Gibbs free energy in terms of $x$ as (Oono, 2017)

$$
\begin{aligned}
& \frac{U}{\epsilon}=\left[\frac{N x^{N}}{x^{N}-1}+\frac{x}{1-x}\right] \\
& <s>=\left[\frac{N x^{N}}{x^{N}-1}+\frac{x}{1-x}\right] \\
& \frac{s}{k}=\left[\frac{N x^{N}}{x^{N}-1}+\frac{x}{1-x}\right] \ln \left[\frac{\Omega}{x}\right]+\ln \left[x^{N}-1\right]-\ln [x-1] \\
& \frac{F}{\epsilon}=\ln \left[\frac{x-1}{x^{N}-1}\right]-\ln \left[\frac{\Omega}{x}\right]
\end{aligned}
$$

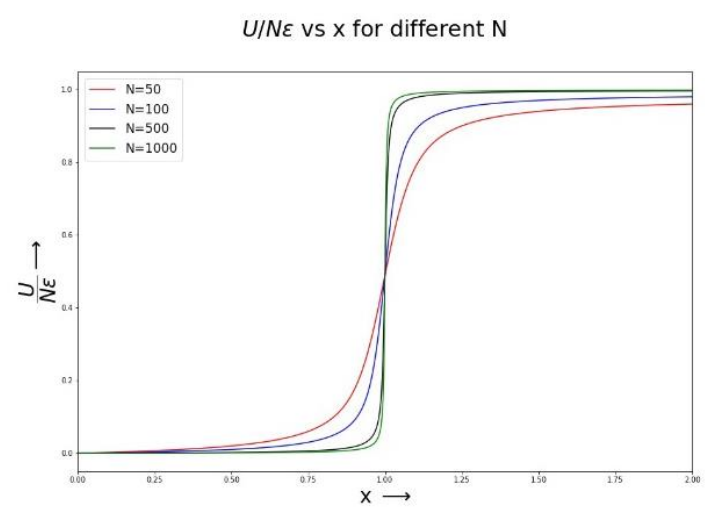

Average number of links per $\mathrm{N}$ vs $\mathrm{x}$ for different $\mathrm{N}$

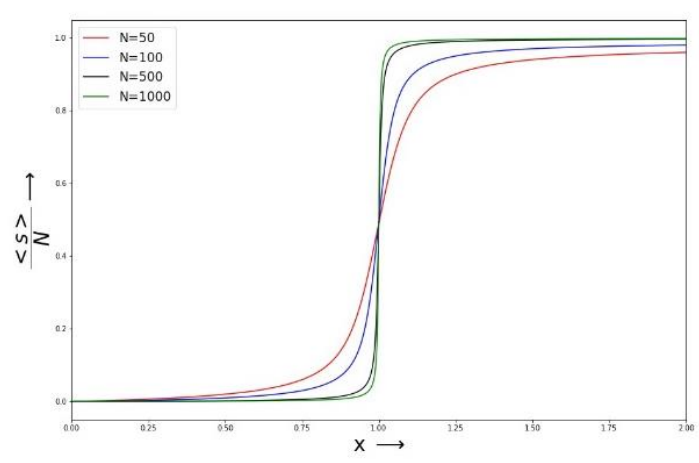

$\mathrm{S} / \mathrm{N} \varepsilon$ vs $\mathrm{x}$ for different $\mathrm{N}$
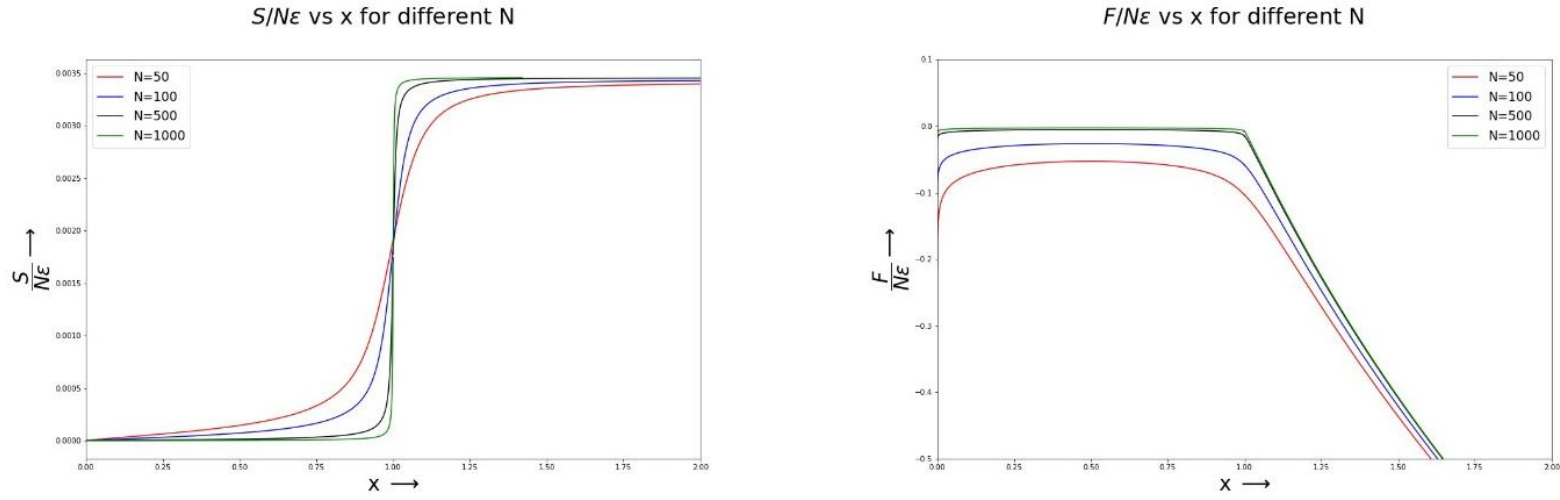

Figure 2. Graphs showing the thermodynamic quantities vs $x$

Something interesting is happening in the limiting case when $x$ approaches one in all the equations. In the Figure 2 below, we plot the values of all the thermodynamic quantities as a function of $x$ at different values of $N$, say $50,100,500,1000$. The discontinuity in the Figure 2 corresponds to a phase change. It looks like the expression has a discontinuity centered at $\mathrm{x}=1$ when $N \rightarrow \infty$. It is true 
that $e^{-\beta \epsilon}$ can never be to equal one, so we will never see a phase transition in the traditional solution to the zipper problem where $\Omega=1$. However, the expression $x=\Omega e^{-\beta \epsilon}$ can be one if $\Omega$ is large enough i.e if $\Omega e^{-\beta \epsilon}=1$ then $\Omega=e^{\frac{\epsilon}{k T_{c}}}$. When the degeneracy of the system is equal to $e^{\frac{\epsilon}{k T_{c}}}$, we will see a phase transition. Rearranging we get $T_{c}=\frac{\epsilon}{k \ln \Omega}$.

\section{Thermodynamics for all temperatures}

We will obtain equations for energy(U), average number of links $(\langle s\rangle)$, entropy(S) and Gibbs free energy for all temperatures (Carvalho-Silva et al., 2020). Only in the case of energy we will give complete derivation.

\section{Average Energy}

We can find the average energy by using the equation (1)

$U=-\frac{\partial}{\partial \beta} \ln \left[\frac{1-\Omega^{N} e^{-\beta N \epsilon}}{1-\Omega e^{-\beta \epsilon}}\right]$

Differentiating we get

$U=\left[-\frac{N \epsilon \Omega^{N} e^{-\beta N \epsilon}}{1-\Omega^{N} e^{-\beta N \epsilon}}\right]+\left[\frac{\epsilon \Omega e^{-\beta \epsilon}}{1-\Omega e^{-\beta \epsilon}}\right]$

Since $\Omega=e^{\frac{\epsilon}{k T_{c}}}$ and $\beta=\frac{1}{k T}$ we get $\Omega e^{-\beta \epsilon}=e^{-\frac{\epsilon}{k T}\left(1-\frac{T}{T_{c}}\right)}$, and hence

$U=\left[\frac{N \epsilon e^{-\frac{\epsilon N}{k T}\left(1-\frac{T}{T_{c}}\right)}}{e^{-\frac{\epsilon N}{k T}\left(1-\frac{T}{T_{c}}\right)}-1}\right]-\left[\frac{\epsilon e^{-\frac{\epsilon}{k T}\left(1-\frac{T}{T_{c}}\right)}}{e^{-\frac{\epsilon}{k T}\left(1-\frac{T}{T_{c}}\right)}-1}\right]$

This is the expression for average energy.

\section{Average Number of Links}

The average number of open links of a zipper is the sum over all states of the probability of being in that state $(P)$ multiplied by the index of the state $(s)$. The number of open links actually represent the free particles which represent the volume of a gas.

$<s>=\sum_{s=0}^{N-1} s P(s)=\sum_{s=0}^{N-1} \frac{s e^{-\beta s \epsilon}}{Q}=\frac{1}{Q} \sum_{s=0}^{N-1} s e^{-\beta s \epsilon}$

where $P(s)=\frac{e^{-\beta s \epsilon}}{Q}$. We can rewrite in terms of a derivative of the partition function and hence the expression for $\left\langle s>\right.$ becomes, $\langle s\rangle=-\frac{1}{\epsilon} \frac{\partial}{\partial \beta} \ln Q_{N}$

Substituting for the partition function we get,

$\left\langle S>=\left[\frac{N e^{-\frac{\epsilon N}{k T}\left(1-\frac{T}{T_{c}}\right)}}{e^{-\frac{\epsilon N}{k T}\left(1-\frac{T}{T_{c}}\right)}-1}\right]-\left[\frac{e^{-\frac{\epsilon}{k T}\left(1-\frac{T}{T_{c}}\right)}}{e^{-\frac{\epsilon}{k T}\left(1-\frac{T}{T_{c}}\right)}-1}\right]\right.$

Entropy

Substituting in the equation (4) and on simplification we get,

$\frac{S}{k}=\frac{\epsilon}{k T}\left[\frac{N e^{-\frac{\epsilon N}{k T}\left(1-\frac{T}{T_{c}}\right)}}{e^{-\frac{\epsilon N}{k T}\left(1-\frac{T}{T_{c}}\right)}-1}-\frac{e^{-\frac{\epsilon}{k T}\left(1-\frac{T}{T_{c}}\right)}}{e^{-\frac{\epsilon}{k T}\left(1-\frac{T}{T_{c}}\right)}-1}\right]+\ln \left[\frac{e^{-\frac{\epsilon N}{k T}\left(1-\frac{T}{T_{c}}\right)}-1}{e^{-\frac{\epsilon}{k T}\left(1-\frac{T}{T_{c}}\right)}-1}\right]$

Helmholtz free energy

Substituting in equation (3) for partition function we get, 
$F=k T \ln \left[\frac{e^{-\frac{\epsilon}{k T}\left(1-\frac{T}{T_{c}}\right)}-1}{e^{-\frac{\epsilon N}{k T}\left(1-\frac{T}{T_{c}}\right)}-1}\right]$

Thermodynamics of the zipper for $T \ll T_{c}, T=T_{c}$ and $T \gg T_{c}$ for large $\mathrm{N}$

In this section we approximate the thermodynamics for extreme conditions (Badasyan, 2021; Dos Anjos, 2019) and also at the phase transition for a large number of links.

Average Energy

In the case of average energy

For $T \ll T_{c}$ substituting for $\ln Q_{N}$ we get

$\frac{U}{N \epsilon}=0$

For $T=T_{C}$ substituting for $\ln Q_{N}$ we get

$U=-N \frac{\partial}{\partial \beta} \ln \left[\Omega\left(1+e^{-\beta \epsilon}\right)\right]$

$U=-\frac{N \Omega e^{-\beta \epsilon}}{\Omega\left(1+e^{-\beta \epsilon}\right)} \times(-\epsilon)=\frac{N \epsilon}{e^{\beta \epsilon}+1}$

$\frac{U}{N \epsilon}=\frac{1}{e^{\beta \epsilon}+1}$

For $\epsilon \rightarrow 0$ we get

$\frac{U}{N \epsilon}=\frac{1}{2}$

For $T \gg T_{c}$ taking the approximation in equation (20) we get

$\frac{U}{\epsilon}=N-1$

For large $\mathrm{N}$

$\frac{U}{\epsilon}=N$

$\frac{U}{N \epsilon}=1$

Equations (25), (29), (32), (33) to (41) beautifully demonstrate phase transition in the zipper model. Carrying out the same procedure as that of average energy for all other thermodynamic quantities we get

Average Number of Links

For $T \ll T_{c}$

$\frac{<s>}{N \epsilon}=0$

For $T=T_{c}$

$\frac{<s>}{N \epsilon}=\frac{1}{2 \epsilon}$

For $T \gg T_{c}$

$\frac{\langle s\rangle}{N \epsilon}=\frac{1}{\epsilon}$

Entropy 
Momentum: Physics Education Journal, 6 (1), 2022, 7

Reshma Perayil, Kandoth Murkoth Udayanandan, Prasanth Pulinchery

For $T \ll T_{c}$

$\frac{S}{N \epsilon}=0$

For $T=T_{c}$

$\frac{S}{N \epsilon}=\frac{1}{2 T_{c}}$

For $T \gg T_{c}$

$\frac{S}{N \epsilon}=\frac{1}{T_{c}}$

Helmholtz free energy

For $T \ll T_{c}$

$\frac{F}{N \epsilon}=0$

For $T=T_{c}$

$\frac{F}{N \epsilon}=0$

For $T \gg T_{c}$

$\frac{F}{N \epsilon}=1-\frac{T}{T_{c}}$

In Figure 3 we plot the thermodynamic quantities vs $\frac{T}{T_{c}}$ which shows the phase transition in terms of the transition temperature.

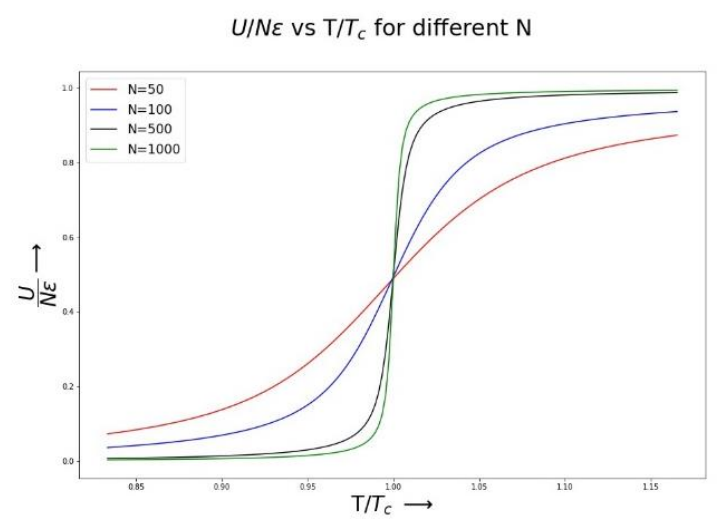

Average number of links per $\mathrm{N}$ vs $T / T_{c}$ for different $\mathrm{N}$
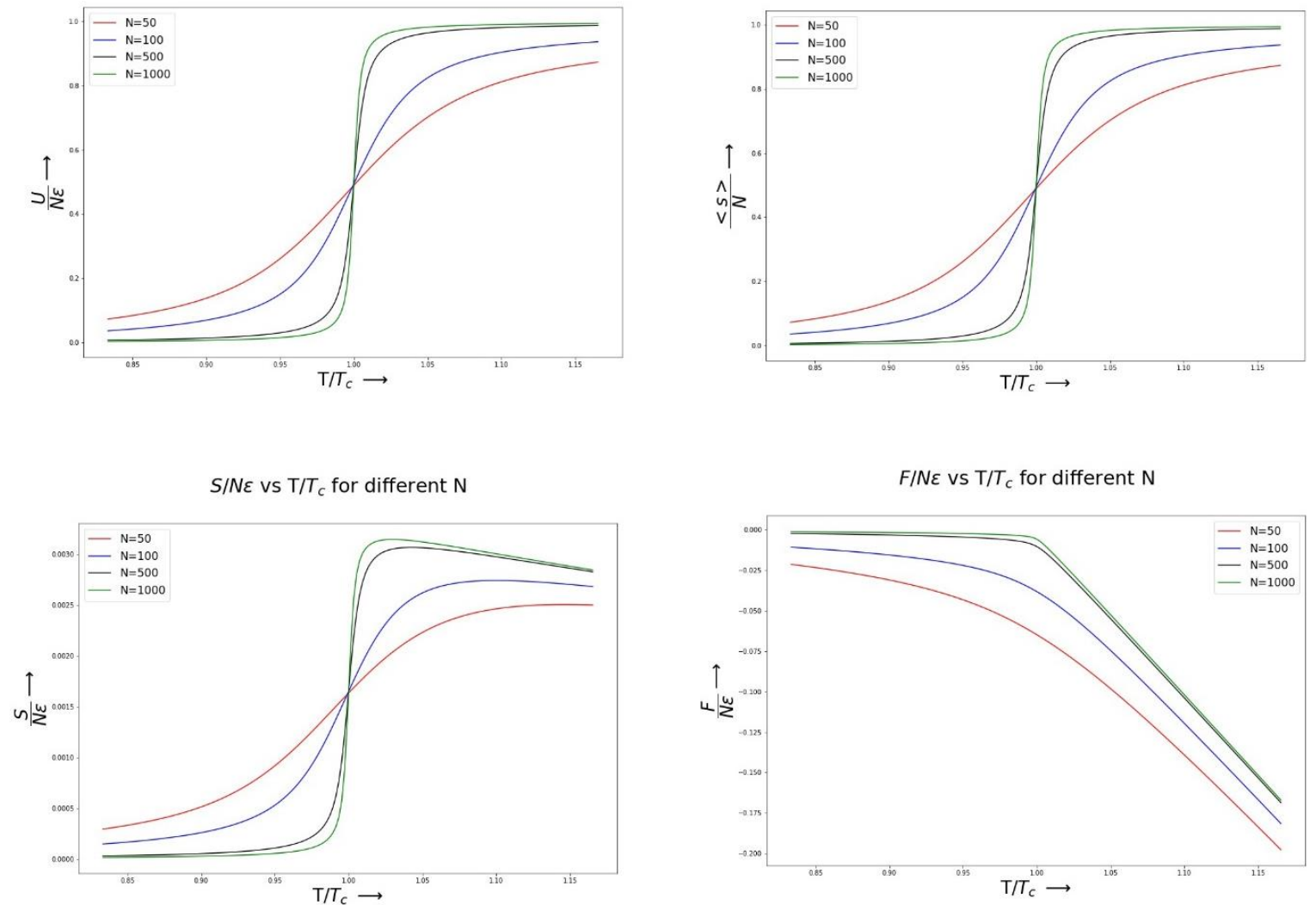

Figure 3. Graph showing the thermodynamic quantities vs $\frac{T}{T_{C}}$ 


\section{Conclusion}

We have established the phase transition in a zipper by finding the thermodynamics in case of quantities like energy, average number of links, entropy, and Gibbs free energy. Except Gibbs free energy, all others showed discontinuity at phase transition temperature, which agrees with theoretical predictions. The opening of links is equivalent to extinction of molecular interactions like liquid getting vaporized. Phase transition is one of the wonders of nature which always attracts physicists' curiosity.

Our paper also shows that proper theoretical modelling will help to reveal the secret behind this phenomenon exhibited by many systems.

\section{Acknowledgment}

Authors wish to thank Mr. Gautham Dathatreyan, MSc Physics, Department of Science, Amrita Vishwa Vidyapeetham, Coimbatore for the plotting using Python.

\section{References}

Ali, M. (2021). Transition energy, orientation force and work done in transitional behavior atoms: formulating new principles in thermodynamics. ChemRxiv, 3. https://doi.org/10.26434/chemrxiv.11553057.v3

Badasyan, A. (2021). System size dependence in the Zimm-Bragg model: partition function limits, transition temperature and interval. Polymers, 13(12), 1985. https://doi.org/10.3390/polym13121985

Bagchi, B. (2018). Statistical mechanics for chemistry and materials science. CRC Press. https://doi.org/10.1201/9781315113951

Banuti, D., Raju, M., Ma, P. C., Ihme, M., \& Hickey, J.-P. (2017). Seven questions about supercritical fluids - towards a new fluid state diagram. 55th AlAA Aerospace Sciences Meeting, 1106. https://doi.org/10.2514/6.2017-1106

Bellantuono, L., Janik, R. A., Jankowski, J., \& Soltanpanahi, H. (2019). Dynamics near a first order phase transition. Journal of High Energy Physics, 2019(10), 146. https://doi.org/10.1007/JHEP10(2019)146

Brahmachari, S., \& Marko, J. F. (2018). DNA mechanics and topology. In Biomechanics in Oncology (pp. 11-39). Springer. https://doi.org/10.1007/978-3-319-95294-9_2

Carvalho-Silva, V. H., Coutinho, N. D., \& Aquilanti, V. (2020). From the kinetic theory of gases to the kinetics of rate processes: On the verge of the thermodynamic and kinetic limits. Molecules, 25(9), 2098. https://doi.org/10.3390/molecules25092098

Deger, A., Brandner, K., \& Flindt, C. (2018). Lee-Yang zeros and large-deviation statistics of a molecular zipper. Physical Review E, 97(1), 012115. https://doi.org/10.1103/PhysRevE.97.012115

Deger, A., \& Flindt, C. (2019). Determination of universal critical exponents using Lee-Yang theory. Physical Review Research, 1(2), 023004. https://doi.org/10.1103/PhysRevResearch.1.023004

Dos Anjos, P. H. R. (2019). Phase transitions and zeros of the partition function: An introduction. Orbital: The Electronic Journal of Chemistry, 11(2). https://doi.org/10.17807/orbital.v11i2.1362

Finkelstein, A. V, \& Ptitsyn, O. (2016). Protein physics: A course of lectures. Elsevier.

Fultz, B. (2020). Phase transitions in materials. Cambridge University Press.

Gridnev, S. A., \& Popov, I. I. (2020). Kinetics of phase transformation at the Curie point of ferroelectric ceramic Ba 0.8 Sr 0.2 TiO 3. Ferroelectrics, 561(1), 127-134. https://doi.org/10.1080/00150193.2020.1736925

Inaba, H. (2015). The development of ensemble theory. The European Physical Journal H, 40(4-5), 489-526. https://doi.org/10.1140/epjh/e2015-60034-2 
Janke, W., Schierz, P., \& Zierenberg, J. (2017). Transition barrier at a first-order phase transition in the canonical and microcanonical ensemble. Journal of Physics: Conference Series, 921(1), 012018. https://doi.org/10.1088/1742-6596/921/1/012018

Kojima, C., Sk, U. H., Fukushima, D., Irie, K., Akazawa, N., Umeda, M., \& Niidome, T. (2015). Effect of main chain conformation on thermosensitivity in elastin-like peptide-grafted polylysine. RSC Advances, 5(127), 104900-104906. https://doi.org/10.1039/C5RA23865J

Koksharov, Y. A. (2020). Analytic solutions of the Weiss mean field equation. Journal of Magnetism and Magnetic Materials, 516, 167179. https://doi.org/10.1016/j.jmmm.2020.167179

Mogliacci, S., Kolbé, I., \& Horowitz, W. A. (2019). Phase transitions in finite size systems. Journal of Physics: Conference Series, 1271(1), 012022. https://doi.org/10.1088/1742$6596 / 1271 / 1 / 012022$

Naiser, T., Kayser, J., Mai, T., Michel, W., \& Ott, A. (2008). Position dependent mismatch discrimination on DNA microarrays - experiments and model. BMC Bioinformatics, 9(1), 509. https://doi.org/10.1186/1471-2105-9-509

Oono, Y. (2017). Perspectives on statistical thermodynamics. Cambridge University Press.

Park, W. M. (2020). Coiled-coils: The molecular zippers that self-assemble protein nanostructures. International Journal of Molecular Sciences, 21(10), 3584. https://doi.org/10.3390/ijms21103584

Sauer, T. (2017). A look back at the Ehrenfest classification. The European Physical Journal Special Topics, 226(4), 539-549. https://doi.org/10.1140/epjst/e2016-60344-y

Shamis, M., \& Zeitouni, O. (2018). The Curie-Weiss model with complex temperature: Phase transitions. Journal of Statistical Physics, 172(2), 569-591. https://doi.org/10.1007/s10955-017$1812-0$

Thiele, U., Frohoff-Hülsmann, T., Engelnkemper, S., Knobloch, E., \& Archer, A. J. (2019). First order phase transitions and the thermodynamic limit. New Journal of Physics, 21(12), 123021. https://doi.org/10.1088/1367-2630/ab5caf

Xiong, S., Qi, W., Huang, B., Wang, M., \& Wei, L. (2011). Gibbs free energy and size-temperature phase diagram of hafnium nanoparticles. The Journal of Physical Chemistry C, 115(21), 1036510369. https://doi.org/10.1021/jp200093a

Zeng, F.-L., Zhou, X.-Y., Li, N., Wang, A.-B., Wang, W.-K., Jin, Z.-Q., Ren, Y.-R., Fang, B.-J., Yuan, N.-Y., \& Ding, J.-N. (2021). A multifunctional zipper-like sulfur electrode enables the stable operation of lithium-sulfur battery through self-healing chemistry. Energy Storage Materials, 34, 755-767. https://doi.org/10.1016/j.ensm.2020.10.025

Zhu, M., Ren, K., Liu, L., Lv, S., Miao, X., Xu, M., \& Song, Z. (2019). Direct observation of partial disorder and zipperlike transition in crystalline phase change materials. Physical Review Materials, 3(3), 033603. https://doi.org/10.1103/PhysRevMaterials.3.033603 